\title{
O USO DA PRÓTESE NA CORREÇÃO DAS HÉRNIAS DA PAREDE ABDOMINAL É UMAVANÇO, MAS O SEU USO INDISCRIMINADO, UM ABUSO
}

\author{
PROSTHESIS USAGE IN ABDOMINAL WALL HERNIA CORRETIONS IS AN ADVANCE, \\ BUT THE INDISCRIMINATE USAGE, IS AN ABUSE
}

\author{
José Guilherme Minossi, TCBC-SP'; \\ Alcino Lázaro da Silva, ECBC-MG ${ }^{2}$; César Tadeu Spadella ${ }^{3}$
}

\section{INTRODUÇÃO}

A hérnia da parede abdominal é uma entidade clínica de elevada prevalência, tanto na população adulta como infantil ${ }^{1}$, estimada entre $3 \%$ a $8 \%$, sendo cinco a seis vezes mais comum no sexo masculino. Cerca de $75 \%$ de todas as hérnias ocorrem na região inguinal. As hérnias incisionais e ventrais representam aproximadamente $10 \%$ de todas as hérnias e as femorais, apenas $3 \%$. As hérnias incomuns são responsáveis por $5 \%$ a $10 \%$ dos casos restantes.

A hérnia e seu reparo cirúrgico são um capítulo extensamente estudado e de profundo interesse por parte dos cirurgiões ${ }^{2}$. As correções estão muito distantes de um consenso entre os cirurgiões, havendo muitas divergências mesmo entre as grandes escolas médicas.

O marco na correção das hérnias deve ser atribuído a Edoardo Bassini (1844 - 1924) que, em 1884, na Itália, lançou as bases consideradas, até recentemente, as mais racionais para o tratamento das hérnias inguinais ${ }^{3}$.

Outros cirurgiões como Marcy (1837 - 1924), discípulo norte americano de Lister, Halsted (1852 - 1922), Mc Vay (1911 - 1987) e outros cirurgiões do final do século 19, e começo do século 20, definiram a anatomia patológica e as principais técnicas operatórias, usadas até hoje.

Mc Vay-Anson marcaram época na evolução do tratamento da herniorrafia inguinocrural por terem utilizado o ligamento iliopectíneo ou de Cooper para ancorar a parede parietal medial. A primeira utilização do ligamento de Cooper, em preferência ao ligamento de Poupart, foi atribuída a Georg Lotheissen (1898).

Coube também a Mc Vay a observação de que as hérnias inguinais indireta volumosa e a direta e a hérnia crural representavam importantíssimos defeitos da parede inguinal posterior. O reparo proposto por este cirurgião, o qual excluía as pequenas hérnias inguinais indiretas, não só foi bem sucedido, como veio a substituir a operação de Bassini. Esta cirurgia recebeu, a seguir, o epônimo de "reparo de Mac Vay" 4 .
A técnica de Shouldice foi, na verdade, um ressurgimento aperfeiçoado do procedimento original de Bassini ${ }^{5}$ : aproximação e sutura, em tripla camada, do músculo oblíquo interno, transverso e fáscia transversal ao ligamento inguinal. Bassini já realizava abertura ampla da fáscia transversal em toda sua extensão, antes de se efetuar a sutura do assoalho inguinal $^{6}$. Shouldice apenas disciplinou a técnica original de Bassini, ao realizar a sutura estratificada em vários planos.

Mais recentemente, se popularizou a abordagem posterior pré-peritoneal das hérnias inguinais, revivida e preconizada por Nyhus (epônimo: operação de Nyhus ${ }^{7}$ ).

Na década de 90, houve modificações revolucionárias no tratamento das hérnias inguinais. A introdução do conceito de herniorrafia com livre tensão deu espaço aos reparos baseados na colocação de telas (Lichtenstein, "Mesh Plug", Nyhus e reparo laparoscópico).

A partir de 1946, após estudos experimentais, foi introduzido na prática médica o uso de próteses metálicas, como o tântalo e, posteriormente, o aço inoxidável. A fragmentação destes materiais, no entanto, com risco de penetração em vasos e nervos, e a eventual migração destes para outras partes do organismo, limitou o uso desta técnica, encorajando pesquisas com outros materiais, como o náilon, o polietileno, o polivinil, o dácron e o téflon. Esses materiais, todavia, associaram-se a um maior índice de infecção e rejeição. O polipropileno monofilamentar, material inerte com baixo índice de infecção e fistulização, é o material preferido pela maioria dos cirurgiões.

As próteses, tanto biológicas como artificiais, foram inicialmente utilizadas para apoiar um reforço realizado pela técnica convencional. O cirurgião, ao não se convencer da resistência do reforço, optava por complementá-lo usando uma prótese. Esta conduta, portanto, era excepcional ${ }^{8}$.

$\mathrm{Na}$ década de 60 , chamava-se à atenção para o risco de recidiva da hérnia, caso a sutura fosse feita sob muita tensão, mesmo com o uso de prótese. Para eliminar a tensão local, Usher et al., 1960, preconizaram a utilização da prótese,

\footnotetext{
1. Professor Assistente; Doutor do Departamento de Cirurgia e Ortopedia da Faculdade de Medicina de Botucatu - Unesp;. Titular do CBCD E SOBRACIL.

2. Professor Titular de Cirurgia do Aparelho Digestivo da Faculdade de Medicina da Universidade Federal de Minas Gerais; Titular do CBCD.

3. Professor Adjunto do Departamento de Cirurgia e Ortopedia da Faculdade de Medicina de Botucatu - Unesp.

Recebido em 27/03/2008

Aceito para publicação em 20/05/2008

Conflito de interesses: nenhum

Fonte de financiamento: nenhuma

Trabalho realizado no Departamento de Cirurgia e Ortopedia da Faculdade de Medicina de Botucatu - Unesp.
} 
em substituição ao assoalho do canal inguinal, como ponte e não reforço ${ }^{9}$. O seu uso foi progressivamente se estendendo, passando a ser indicado sempre que o enfraquecimento do assoalho do canal inguinal estivesse presente ${ }^{10}$.

Mais recentemente, com o objetivo de propiciar ao paciente a menor dor pós-operatória e o seu retorno precoce às atividades, o uso de próteses tem sido quase que sistemático por muitos autores ${ }^{11}$.

Há pouco mais de vinte anos, a correção das hérnias inguinais também passou a ser feita por videolaparoscopia. Diversos tipos de procedimentos foram descritos, como o fechamento simples do anel inguinal interno nas hérnias indire$\operatorname{tas}^{12}$ e a oclusão do anel inguinal interno com tampão ("plug") de tela ${ }^{13}$. Foram abandonados devido ao elevado índice de recidiva ${ }^{14}$, muito superior à correção pela via convencional, cujos índices não ultrapassam $2 \%$.

Posteriormente, procedeu-se à colocação de uma tela de polipropileno para reforçar o assoalho inguinal, tanto pela via transabdominal (TAPP) como por acesso extraperitoneal (TEP), que são os procedimentos mais utilizados no momento ${ }^{15}$. A análise crítica deste novo método necessita de um tempo mais longo de observação.

Inovando o tratamento cirúrgico das hérnias inguinais o saco herniário foi melhor estudado em laboratório, onde se mostrou que o mesmo é rico em musculatura lisa e colágeno, podendo, por isso, ser usado como reforço durante as intervenções, com as vantagens de não acarretar reação do tipo corpo estranho e nem aumentar o índice de complicações locais ${ }^{16-18}$.

Como se pode observar, o tratamento cirúrgico das hérnias, particularmente das hérnias inguinais, foi e continua sendo um desafio para o cirurgião e a recidiva um fato tão certo quanto a própria existência da hérnia, obviamente, motivo de grande frustração para pacientes e cirurgiões. Não se pode dizer que haja um consenso a respeito da melhor técnica a ser utilizada nos diferentes defeitos herniários que acometem a região inguinal. O que observamos é a existência de um grande número de técnicas e opiniões contraditórias, as quais justificam plenamente, tanto a procura de novos métodos para o tratamento desta afecção, como a publicação de casuísticas individuais ou de grupos que lidam com as operações da parede abdominal.

\section{Complicações das Próteses}

Um biomaterial ideal deveria ter as seguintes características: resistência mecânica semelhante a dos tecidos regionais; não propiciar infecção, o que implicaria na sua retirada; ser rapidamente incorporado pelo organismo; não estimular a fibrose excessiva; não promover reações de corpo estranho; não ser carcinogênico; ser esterilizável, sem alteração de suas qualidades e ser economicamente viável. Infelizmente nenhum dos materiais existentes até o momento, preenche todos os requisitos ${ }^{19,20}$

As complicações possíveis com o uso de próteses são: infecção, rejeição do material, deformação da prótese, formação de coleções serosas e sangüíneas, aderências peritoneais, fistulização em vísceras ôcas, alterações da espermatogênese, formação de tumor (carcinogênese), e inguinodinia.

\section{Infecção}

A prótese, como um material sintético estranho ao organismo, tem a capacidade de agravar, potencializar ou prolongar a infecção ${ }^{21}$.

Admite-se que as telas monofilamentares (como a de polipropileno) tolerariam melhor a infecção do que as multifilamentares (como a de poliéster). Com a tela de politetrafluoroetileno expandido (PTEFe) a infecção é de mais difícil controle. A sua arquitetura interna apresenta fendas e poros nos quais as bactérias podem se multiplicar. Os neutrófilos, com diâmetros superiores aos das bactérias, não conseguem chegar a estes patógenos que se escondem em seus orifícios ${ }^{22}$

Nas hérnias incisionais, as correções com próteses apresentam índices de infecção de parede que variam entre $6 \%$ a $12 \%$; índices estes maiores que os encontrados nos reparos sem prótese $(3 \%)$.

\section{Rejeição}

É uma complicação rara que ocorre, na maioria dos casos, associada à infecção. Uma prótese não incorporada ou rejeitada habitualmente é envolvida em secreção estéril. Há reação de corpo estranho, formada por células gigantes. Coleções líquidas encistadas podem se manifestar na pele cicatrizada e no sítio da cicatriz cirúrgica. Com o tempo, a coleção líquida drena para a pele, através de um "sinus", com eliminação de líquido seroso, que posteriormente torna-se purulento, podendo haver exposição parcial ou total da prótese, caracterizando a extrusão $0^{20,23}$.

\section{Deformação da Prótese}

As próteses de polipropileno não fixadas costumam se deformar pela retração do tecido cicatricial. As margens da tela se curvam e se enrolam sobre si mesmas, razão pela qual sempre devem ser fixadas ou colocadas entre duas camadas mioaponeuróticas ${ }^{24}$. Já a tela multifilamentar de poliéster, por ser flexível e se adaptar com facilidade aos tecidos, não necessita ser fixada, como demonstra Stoppa $(1995)^{25}$. As próteses de PTEFe, independentemente de seu tamanho, devem ser fixadas, pois sua integração é demorada ${ }^{26}$.

\section{Coleções serosas ou sangüíneas}

$\mathrm{O}$ acúmulo de coleções serosas ou sangüíneas ao redor das telas é observado com freqüência, especialmente quando as próteses são de grandes dimensões e as áreas de descolamento extensas. Estes líquidos retardam a sua integração e impedem a chegada de hematócitos e de antibióticos, funcionando como meio de cultura. Com o passar do tempo, é relativamente comum que estas coleções serosas e assépticas se tornem purulentas. Nestes casos, a retirada total ou parcial das partes seqüestradas da prótese, frequientemente é necessária, pois é pouco provável que a integração da prótese tenha ocorrido ${ }^{27}$.

Da mesma forma que as próteses planas, que estimulam a fibroplasia e a incorporação tecidual, os tampões ("plugs"), sejam maciços ou em forma de cones, podem também criar, ao seu redor, um espaço preenchido por serosidade. $\mathrm{Na}$ melhor das hipóteses, estas coleções permanecem esté- 
reis, cuja evolução é incerta mas que, meses ou anos após, sofrem o mesmo processo já descrito, vindo a se exteriorizar pela cicatrização cirúrgica, através de trajetos fistulosos, que se mantêm enquanto persistir a presença do corpo estranho ${ }^{28}$.

\section{Aderências e fistulização em vísceras ocas}

O processo inflamatório provocado pela prótese promove aderência aos tecidos circunjacentes ${ }^{23}$, podendo levar à obstrução intestinal $^{29}$, granuloma espermático ${ }^{30}$ e a formação de fístulas intestinais ${ }^{31,32}$. O primeiro estágio para a formação de uma fístula é representado pela aderência do material sintético à víscera adjacente ${ }^{22}$.

Nos métodos que utilizam o acesso pré-peritoneal, seja este realizado por via aberta ou laparoscópica, caso o processo inflamatório se propague até as estruturas viscerais, como o intestino delgado, o cólon ou a bexiga urinária, o mesmo provocará, inicialmente, aderências que mais tarde evoluirão com erosão das paredes viscerais e fistulização ${ }^{32-34}$.

A fragmentação da prótese pode ocorrer por causa da recorrência da hérnia, ou devido à obesidade e ao esforço físico do paciente, levando à tensão que excede a resistência da tela em sua porção central, no reparo da hérnia incisional ${ }^{35-}$ ${ }^{37}$. Um fragmento da prótese provoca um abscesso e a subseqüente fístula ${ }^{38}$. $\mathrm{O}$ risco de fistulização não desaparece com o tempo, podendo se manifestar entre 14 a 20 anos após a cirurgia $^{39,40}$

A incidência de aderências e fistulizações varia de $0,3 \%$ a $23 \%$ quando se usa a tela de polipropileno ${ }^{41}$.

As próteses de PTEFe apresentam menor reação tecidual, mas na prática também determinam aderências, embora em menor quantidade ${ }^{21}$.

A prótese inabsorvível, que contém uma camada absorvível em contato com o peritônio, para evitar aderências, é ineficaz, pois as mesmas se manifestam logo após a sua completa absorção ${ }^{22}$.

\section{Alterações da espermatogênese}

Existe no momento um estudo experimental que mostra que a tela de polipropileno em contato com o funículo espermático de cães provoca alterações histológicas com redução da espermatogênese ${ }^{42}$.

Clinicamente, oito instituições americanas reportaram um total de 14 casos de azoospermia secundária à obstrução do deferente por tela de polipropileno ${ }^{43}$.

\section{Formação de tumor (carcinogênese)}

O destino em longo prazo das próteses e suas interrelações com os tecidos circunjacentes é motivo de preocupação para muitos estudiosos do assunto ${ }^{19,44}$. Nyhus e Langer et al. alertaram para o risco em potencial de a persistente reação tecidual desencadear a transformação tumoral maligna local - fato observado em experimentos animais ${ }^{45,46,41}$.

\section{Inguinodinia}

A dor inguinal crônica após herniorrafia inguinal tem sido descrita como uma nova síndrome: "inguinodinia por tela" ${ }^{47}$. Embora a maioria dos casos ocorra em pacientes que receberam prótese, a inguinodinia também pode acontecer nas operações convencionais, devendo-se, portanto, ser evitado o termo "inguinodinia por tela".

Ela decorre da presença de pontos, clipes, prótese junto ao tubérculo púbico (dor somática) ou devido à lesão dos nervos sensitivos da região inguinal (dor neuropática). Esta última pode ser primária, isto é, decorrente do trauma operatório, por secção parcial, estiramento, esmagamento, eletrocoagulação ou compressão do nervo. É secundária quando há envolvimento por cicatriz ou irritação por granuloma. As próteses sintéticas, de um modo geral, estimulam a fibroplasia de uma forma desorganizada, envolvendo estruturas que, provavelmente, após a sua retração, provocam a compressão cicatricial de nervos sensitivos.

É importante definir-se o tipo de dor, se neuropática (neurálgica) ou somática, pois têm fisiopatologia e tratamento diferentes.

A freqüência da inguinodinia é muito variável na literatura, de 0 a $37 \%{ }^{48,49}$, e atinge marcas alarmantes nas correções cirúrgicas livres de tensão que usam prótese sintética ${ }^{45}$. A restrição das atividades, física ou esportiva pode chegar a $18 \%$ dos $\operatorname{casos}^{50,51}$.

A inguinodinia parece ocorrer numa freqüência um pouco menor nas operações por via laparoscópica, estimada em $2 \%$ a $2,5 \%$ dos reparos ${ }^{52-54}$, do que naquelas realizadas por via anterior, de 2,1 a $37 \%{ }^{49,55-57}$.

Pelo fato desta síndrome ser potencialmente incapacitante e de difícil tratamento, a sua prevenção merece cuidado especial ${ }^{58}$. Uma série de medidas deve ser utilizada $^{59}$, inclusive o uso criterioso de próteses, que embora não seja a causa exclusiva desta condição, dificulta em muito um eventual tratamento cirúrgico da dor crônica, uma vez que o uso de próteses aumenta a fibrose e a aderência dos tecidos.

\section{Hérnias Inguinais}

Achamos que o passo inicial mais importante no tratamento das hérnias inguinais é a adoção de uma classificação. Se bem reconhecida, poderia funcionar tanto como orientação para a dissecção do canal inguinal e femoral, como também na avaliação dos vários reparos cirúrgicos e também diferenciar claramente os problemas anatômicos existentes.

Em 1967, Casten apresentou um esquema de classificação das hérnias inguinocrurais baseado em sua anatomia funcional e tratamento operatório ${ }^{60}$.

Em 1970, Halverson e McVay classificaram as hérnias em quatro grupos com base em defeitos anatômicos e nas técnicas de reparo ${ }^{61}$.

Nyhus descreveu um esquema de classificação que se baseia na anatomia do espaço pré-peritoneal, utilizando como critérios, além do anatômico, o tamanho do anel herniário e as condições da parede posterior do canal inguinal ${ }^{62}$.

Em 1986, Gilbert estabeleceu um esquema de classificação com base nos defeitos anatômicos e funcionais identificados no intraoperatório ${ }^{63}$. Os tipos 1,2 e 3 são indiretos, enquanto os tipos 4 e 5 são diretos. Rutkow e Robbin, em 1993, acrescentaram um tipo 6 para as hérnias mistas, que apresentam um componente tanto direto, como indireto, e o tipo 7 para as hérnias femorais ${ }^{64}$. 
O tipo 1 possui um anel interno de diâmetro normal (não superior a $2 \mathrm{~cm}$ ), através do qual passa um saco peritoneal de qualquer tamanho. $\mathrm{O}$ tipo 2 possui um anel interno moderadamente aumentado, que não mede mais que $4 \mathrm{~cm}$. $\mathrm{O}$ tipo 3 possui um anel interno aumentado com mais de $4 \mathrm{~cm}$, sendo que o saco desliza freqüentemente para o escroto e os músculos oblíquo interno e transverso estão deslocados superiormente, expondo a fáscia transversal. No tipo 4, todo o assoalho do canal iguinal é defeituoso (hérnia direta de colo largo). $\mathrm{O}$ tipo 5 apresenta um defeito diverticular da parede posterior do canal inguinal.

Preferimos a classificação de Gilbert por ser mais didática e incluir as hérnias mistas e os defeitos diverticulares, não contemplados na classificação de Nyhus. A crítica à classificação de Gilbert seria o fato de não incluir as hérnias recidivadas, que podem ser classificadas à parte ou num grupo 8 .

Procuramos obedecer ao conceito de que para cada tipo de hérnia deve ser realizado um tipo de correção, conduta que também é compartilhada por outros serviços ${ }^{65,66}$, evitando-se o tratamento único para diferentes defeitos ${ }^{67}$.

Entendemos que os defeitos herniários do tipo 1 e 2 de Gilbert devam ser tratados por técnicas convencionais, sem o uso de prótese, sendo de fundamental importância a ligadura alta do saco herniário, junto ao anel inguinal profundo.

Nas hérnias tipo 1, isto é, indiretas com anel interno normal, não há motivo para a operação progredir além do tratamento do saco herniário, onde este procedimento leva à cura sem nenhuma recidiva em nossa casuística ${ }^{55}$.

Quando a hérnia é indireta, com pequeno aumento do anel inguinal interno (Gilbert 2), sem defeito posterior, o tratamento do saco e um reforço pela técnica de Bassini modificado apresentou $1,8 \%$ de recidiva ${ }^{55}$.

Vários outros estudos demonstram a eficácia de técnicas convencionais quanto ao índice de recidivas e outras complicações. Kingsnorth et al., após 24 meses de seguimento, observaram sete recidivas $(3,1 \%)$ pela técnica de Shouldice, e quatro $(1,7 \%)$ nos operados pela técnica de Bassini modificada $^{68}$.

Kuy et al., pelo contrário, comparando as técnicas de Bassini e de Shouldice modificada (dois planos apenas) em um grande número de casos, concluíram que o índice de recidiva após dois anos era de $8,7 \%$ no primeiro grupo e de $2,3 \%$ no segundo ${ }^{69}$. Outro estudo também casualizado, comparando essas duas técnicas, foi realizado por Paul et al., que após seguimento médio de 3,3 anos, obtiveram um resultado que favorece a operação pela técnica de Shouldice, que apresentou índice de recidiva de $1,7 \%$ contra $9,6 \%$ na operação de Bassini modificada ${ }^{70}$.

A comparação das técnicas de Bassini, Shouldice e de Lotheinsen - Mc Vay foi realizada por Mittelstaedt et al. Em estudo randomizado, com 119 pacientes portadores de 136 hérnias, divididos em grupos equivalentes quanto ao número e tipo de hérnias (diretas ou indiretas), obtiveram taxas de recidivas projetadas (teste de Kaplan-Mayer) de 35,7\% para a técnica de Bassini, 23,7\% para a de Shouldice e $8,5 \%$ para a de Mc Vay. A incidência elevada na técnica de Bassini variou com o tipo de hérnia ( $29 \%$ nas diretas e $16 \%$ nas indiretas) e levou os autores a interromper o estudo com esta técnica ${ }^{71}$ Os índices de recidiva com as técnicas de Shouldice e de Mc Vay, projetadas atuarialmente para quatro anos, foram, respectivamente, de $23,7 \%$ e $8,5 \%$.

O que chama à atenção é a não classificação dos tipos de hérnias e, conseqüentemente, a aplicação de uma técnica muitas vezes não adequada ao tipo de defeito, como por exemplo, tratar as hérnias diretas ou indiretas grandes com a operação de Bassini, em que certamente a recidiva atingirá índices proibitivos.

Em nossa casuística observamos que alguns pacientes com hérnias dos tipos 3, 4, 5 e 6 de Gilbert foram eventualmente tratados pela técnica de Bassini, procedimento não recomendado para este defeito. Como esperado, o índice de recidiva nestes casos foi elevado $(41 \%)$. Este tipo de defeito, em nossa padronização, deveria ter sido tratado pela técnica de Mc Vay ou com o uso de tela de polipropileno, conforme descrito por Falci-Lichtenstein. Em nossa experiência, critérios para o uso ou não de prótese foram baseados principalmente nas condições das estruturas anatômicas, tais como: boa consistência da área conjunta (arco aponeurótico do transverso) com inserção no arco púbico, triângulo inguinal de dimensões não muito aumentadas e fáscia transversal de consistência razoável. Nessas condições, optou-se pela técnica convencional, caso contrário foi utilizada a prótese. Em 253 hernioplastias realizadas pela técnica de Mc Vay, 24 apresentaram recidiva (9,5\%). Em 95 casos de defeitos posteriores e de hérnias recidivadas a preferência foi pela utilização de prótese de polipropileno. Nesses casos observamos apenas dois casos de recidiva $(2,1 \%)^{55}$.

Früs e Lindahl (1996) realizaram um estudo casualizado comparando a técnica sem tensão de Lichtenstein, realizada em hérnias diretas e indiretas, com a de Mc Vay, em hérnias diretas. Após seguimento de dois anos, observaram índices de recidiva em $7 \%$ nas hérnias diretas pela técnica de Falci-Lichtenstein e de 30\% nas de Mc Vay. O elevado número de recidivas após a operação tipo Falci-Lichtenstein (7\%) e, principalmente, após a operação de Mc Vay (30\%), superiores aos índices relatados na literatura, foi atribuído ao fato de as cirurgias serem realizadas por cirurgiões em fase de treinamento ${ }^{72}$. Em nosso serviço, por se tratar de hospital universitário, o nosso índice de recidiva acha-se um pouco mais elevado que o habitual, principalmente por se manifestar já no primeiro ano de correção (57\% das recidivas ocorreram nesse período), sugerindo serem decorrentes de erros técnicos. Este fato tem sido observado em inúmeras outras casuísticas ${ }^{73,74}$.

O nosso estudo mostra que para as hérnias inguinais com anel interno normal (tipo 1), não há motivos para que o tratamento se estenda além do tratamento do saco herniário, pois a recidiva foi nula. Nas hérnias indiretas pequenas, em que o anel inguinal interno encontra-se pouco aumentado, e a anatomia da parede posterior do canal inguinal encontra-se preservada, o tratamento pode ser feito de maneira muito conservadora, com correções convencionais como a operação de Bassini clássica ou modificada, ou ainda com outro tipo de reforço que o cirurgião esteja mais familiarizado. Deve-se evitar, nesses casos, a utilização de técnicas de correção, como 
Mc Vay e Shouldice, as quais, além de serem mais elaboradas, têm execução mais difícil, alteram mais intensamente a fáscia transversal, uma estrutura importante que se encontra normal nesses tipos de defeitos. Não seria indicado o uso de prótese, bem como a abordagem laparoscópica nessas circunstâncias, principalmente pelo maior custo e alguns inconvenientes, como aumento do risco de infecção, rejeição, aderências, obstruções intestinais, fistulização e inguinodinia ${ }^{75-77}$.

Achamos também que as mulheres, pelo fato de apresentarem, na maioria das vezes, hérnias indiretas, e possuírem a fáscia transversal mais firme e resistente, devam se beneficiar de uma conduta mais conservadora.

Certamente, as maiores dificuldades que o cirurgião enfrenta ficam por conta das hérnias inguinais indiretas grandes, diretas e mistas. Nestes tipos de hérnias, fatores anatômicos, como a inserção alta dos músculos oblíquo interno e transverso, que ocasionam um aumento da área do trígono inguinal (Hessert), associados a fatores biológicos, como o menor conteúdo de colágeno e de fibras elásticas na fáscia transversal, podem comprometer o êxito do procedimento cirúrgico, quando não muito bem analisados.

Nessas eventualidades o cirurgião deve escolher uma entre as diversas técnicas convencionais que ofereçam um bom reforço posterior, como as técnicas de Shouldice e Mc Vay, ou então optar pela utilização de uma prótese, individualizando assim, a correção das hérnias inguino-crurais ${ }^{78-80}$.

Esta conduta individualizada encontra respaldo na literatura médica, principalmente por ser o procedimento preconizado por vários autores (Falci, Lázado da Silva, Silva, Melo, Nyhus) $)^{79,80-83}$.

Outro aspecto é a tendência atual ao uso indiscriminado de prótese nas correções das hérnias inguinais, já que se tem criado uma jurisprudência perigosa para a atividade médica: a de que o uso desses materiais é o tratamento padrão. Neste caso, uma eventual recidiva da hérnia, sem o uso deste material sintético, poderia levar o médico a responder legalmente por este ato ${ }^{84}$. Há que se considerar, ainda, a influência insistente da indústria fabricante desses materiais, durante a comercialização dos seus produtos, nem sempre acompanhados de uma avaliação criteriosa do custo, risco e benefício para os pacientes. Lamentavelmente, porém, em muitos casos, com a conivência pretensiosa do médico.

A influência da indústria pode ser observada, por exemplo, através da Portaria Ministerial nº 2036/GM de 04 de novembro de 2002, que aprova e define valores dos procedimentos da tabela de órteses, próteses e materiais especiais, sob o código 93481390. Esta portaria autoriza o uso de "tela inorgânica de polipropileno com sistema duplo" para o procedimento "herniorrafia inguinal unilateral", sob o código 33011117.

O SUS remunera a indústria produtora do material em $\mathrm{R} \$ 260,00$, o hospital em $\mathrm{R} \$ 217,12$, o cirurgião em $\mathrm{R} \$ 65,67$, o anestesista em R \$36,44 e o auxiliar em R \$ 19,34. Percebe-se que o cirurgião está sendo considerado mais um técnico, que aplica o produto, do que propriamente um profissional que executa um ato altamente qualificado e de grande responsabilidade.

\section{Hérnias Incisionais}

A hérnia incisional, também chamada de eventração, laparocele e hérnia pós-operatória ou hérnia cicatricial, consiste na protrusão de vísceras abdominais, através de orifícios ou áreas da parede abdominal, anormalmente enfraquecidos por traumas e incisões cirúrgicas. Estes defeitos da parede abdominal são de natureza iatrogênica, diferindo das demais hérnias parietais, por não ocorrerem em pontos anatômicos débeis e naturais da parede do abdome. Ocorre em parede íntegra que se torna enfraquecida pela realização de incisões cirúrgicas. Ela representa, portanto, a falência da via de acesso empregada ou a síntese defeituosa dos planos anatômicos parietais, pressupondo, assim, a existência de uma lesão local, ou geral, que compromete a cicatrização ${ }^{85,86}$.

A maioria dos cirurgiões prefere a linha mediana para adentrar à cavidade abdominal, por ser o acesso mais rápido, menos hemorrágico e pela facilidade de ampliações. No segmento supra-umbilical, entretanto, a secção longitudinal da linha branca, condiciona maior incidência de eviscerações pósoperatórias e de eventrações, pois nela se concentram as linhas de forças resultantes da contração dos músculos largos do abdome. Esses músculos tracionam lateralmente a linha branca para ambos os lados, exercendo apreciável força de separação nas bordas da sutura aí praticada.

Sendo a linha branca supra-umbilical uma lâmina aponeurótica com irrigação sangüínea pobre, a cicatrização é demorada e precária; as fibras aponeuróticas, embora entrecruzadas, se deixam cortar ou cedem à penetração da agulha e dos fios pelo insuficiente apoio que oferecem.

Assim, a linha mediana supra-umbilical não se constitui em boa localização para a realização de uma incisão abdominal, pois é um local submetido à maior tensão, com apoio inseguro, oferecendo ainda más condições para a cicatrização ${ }^{87}$.

Já a linha mediana infra-umbilical, onde as forças que atuam são mais fracas devido ao apoio lateral das cristas ilíacas e da arcada crural, se constitui em um bom local para se praticar uma incisão cirúrgica. Há entre os músculos retos uma separação linear e não laminar, podendo a reconstituição da parede ser feita em três planos: na profundidade, o plano peritônio-aponeurótico (arcada de Douglas); no meio, o plano muscular, e na superfície a aponeurose de inserção dos músculos largos, bem vascularizada e firme.

É aceitável a incisão mediana infra-umbilical e condenável a incisão supra-umbilical; ao menos que nesta se proceda a abertura longitudinal sistemática das bainhas dos músculos retos abdominais, e se faça a reconstituição da parede em três planos. Dessa maneira, embora a incisão tenha se localizado na linha de maior tensão abdominal, os inconvenientes já referidos seriam compensados com a reconstituição reforçada em três planos.

Devem ser proscritas as incisões longitudinais pararretais externas ou laterais e, também, as subcostais, pois as mesmas seccionam os nervos intercostais, levando à desnervação dos músculos retos do abdome e ao enfraquecimento da parede abdominal. Essa complicação tem sido denominada ventre de abade ${ }^{88}$. 
As incisões paramedianas pararretais internas e as transversais são as melhores vias de acesso ao abdome, principalmente pelo maior respeito à anatomia e fisiologia da parede, assim como pelo menor número de eviscerações e eventrações.

Percebe-se, portanto, que a prevenção de possíveis eventrações da parede abdominal inicia-se com a escolha de uma incisão abdominal que respeite, ao máximo, a anatomia e a fisiologia da parede abdominal.

Esses princípios anatômicos da parede abdominal, ao nosso ver, podem e devem ser sempre usados por ocasião da correção das hérnias da parede abdominal, particularmente das eventrações.

As hérnias do andar superior do abdome apresentam uma maior dificuldade técnica do que as localizadas no andar inferior. As hérnias supra-umbilicais, de um modo geral, apresentam grandes separações dos músculos retos, particularmente devido a maior tensão na linha média, como já mencionado. Superiormente temos o gradil costal, que de um modo geral dificulta a correção desses defeitos, tanto por técnicas convencionais, quanto os realizados com a utilização de próteses.

Já nas hérnias infra-umbilicais, pela menor tensão da linha média, e pela possibilidade de se fechar a parede em três planos, torna-se mais fácil a correção dos defeitos herniários com a simples reconstituição anatômica da parede, desde que o defeito não seja excessivamente grande, e que o conteúdo do saco herniário seja possível de ser reduzido com facilidade para a cavidade abdominal.

Sendo assim, na correção das hérnias incisionais pode-se indicar inúmeros procedimentos cirúrgicos sem a utilização de próteses, particularmente naqueles com defeitos herniários pequenos e médios (diâmetro do defeito até $6 \mathrm{~cm})$. Nas hérnias grandes $(=10 \mathrm{~cm})$, gigantes $(=20 \mathrm{~cm})$ e monstruosas $(>20 \mathrm{~cm})$, o cirurgião tem ainda a opção de realizar uma transposição peritônio-aponeurótica, técnica de correção desses defeitos que dispensa a prótese e com bons resultados tardios, além de respeitar a fisiologia da parede abdominal ${ }^{88,89}$.
A utilização de prótese na correção das eventrações deveria se restringir aos casos em que não foi possível a reconstrução anatômica da parede abdominal sem tensão, e nas situações em que o saco herniário não pôde ser usado como reforço.

Com base na experiência pessoal dos autores e na revisão da literatura, é possível concluir que:

$1^{\circ}$ Embora as próteses se constituam em uma boa opção para corrigir hérnias da parede abdominal, principalmente pelo fato de poderem ser aplicadas quando não se consegue reconstituir o defeito de maneira anatômica, e sem tensão, elas podem determinar uma série de complicações, tais como: infecção, rejeição, deformação, coleções serosas ou sangüíneas, aderências e fistulização em vísceras ocas, alterações da espermatogênese, carcinogênese e dores crônicas na região inguinal (inguinodinia), o que faz com que o seu uso deva ser criterioso.

$2^{\circ}$ ) Complicações como extrusão crônica da prótese e inguinodinia têm-se constituído em uma situação perturbadora para o paciente e freqüentemente de difícil tratamento.

$\left.3^{\circ}\right)$ Os indícios recentemente aventados da possibilidade de alterações da espermatogênese e carcinogênese, fazem com que a indicação deste material seja muito cautelosa, principalmente em jovens.

$4^{\circ}$ ) Não existe motivo para se utilizar próteses nas hérnias indiretas pequenas (tipo 1 e 2 de Gilbert), em que a simples ligadura do saco e o reforço à Bassini, respectivamente, apresentam recidiva nula ou muito baixa $(<1 \%)$.

Da mesma maneira, a maioria das mulheres com hérnia inguinal não necessitam de prótese, pois as mesmas apresentam fáscia transversal firme e resistente, e quase que exclusivamente são portadoras de hérnias indiretas.

$5^{\circ}$ ) Com relação às hérnias incisionais e as demais hérnias da parede abdominal, a sua correção deve ser baseada na compreensão da fisiologia e da anatomia da parede, não somente em seu tamanho, sendo a técnica a ser utilizada variável, indo desde a simples síntese da parede por planos, utilização do saco em grandes hérnias e, eventualmente, o uso de prótese sintética.

\begin{abstract}
The objective is to alert the surgeon about the indiscriminate use of synthetic prosthesis in the correction of inguinal and incisional hernias. The authors provide a brief history of surgery on hernias and a review of the literature, showing the importance of classifying inguinal hernias to fit the type of surgical correction with the defect found, abstaining from treating all hernias, with the same type of surgical procedure. In our opinion, small indirect inguinal hernias (type 1 and 2 of Gilbert) and hernias in women must not, in general, be treated with prostheses. The synthetic material should be reserved for direct and large indirect hernias. Even so, this attitude, besides determining a higher cost for the procedure, can lead to important complications such as infection, rejection, fistula formation, chronic pain, alterations in spermatogenesis and the possibility of carcinogenesis, according to more recent reports. The physiology and anatomy of the abdominal wall should be considered when dealing with incisional hernia corrections, where the surgeon can choose among many techniques to correct those defects, and in selected cases, utilize synthetic material. We conclude that although the use of biomaterials has constituted a great advance in surgery for abdominal wall hernia corrections because they decrease recurrences, and permit treatment of large abdominal hernias, the indiscriminate prosthesis usage is an abuse, and it can determine many serious complications, certainly avoidable with a well indicated non mesh technique (Rev. Col. Bras. Cir. 2008; 35(6): 416-424).
\end{abstract}

Key words: Abdominal wall; Surgical mesh/complications; Hernia, ventral; Prostheses and implants/utilization. 


\section{REFERÊNCIAS}

1. Ayala LA, Belloso RM, Souchon E, Zavaleta DE. "Hérnias". In: Romero Torres R, editor. Tratado de cirurgia. $2^{\mathrm{a}}$ ed. México: Interamericana: Mc Graw-Hill: 1993. p. 1108.

2. Restrepo JFP. "Hérnias abdominais". In: Coelho JCV editor. Aparelho digestivo-clínico e cirurgia. $3^{\mathrm{a}}$ ed. Rio de Janeiro: Atheneu; 2005. p. 1951-6.

3. Bassini E. Nuovo metodo per la cura radicale dell'ernia. Attilio Catterina: Assoc Med Ital; 1887.

4. Mc Vay CB. Inguinal and femoral hernioplasty: anatomic repair. Arch Surg. 1948; 57(4):524-30.

5. Barroetavena JL, Herzagel. Bassini versus Shouldice. Revisión histórica-bibliográfica. Rev Argent Cir. 1991;60(1):97.

6. Wantz GE. The operation of Bassini as described by Attilio Catterina. Surg Gynecol Obstet. 1989; 168(1):67-80.

7. Patino Restrepo JF, García-Herreros LG, Zundel N, Supelano G, Baptiste de Arrouo S. Hernioplastia preperitoneal com prótesis. Rev Colomb Cirurgia. 1992; 7(2):74-80.

8. Speranzini MB, Deutsch CR. Correção das hérnias inguinais e crurais por via anterior (convencional e com prótese). In:

.Tratamento cirúrgico das hérnias das regiões inguinal e crural. Estado atual. São Paulo: Atheneu; 2001. p.69-100.

9. Usher FC, Cogan JE, Lowry TI. A new technique for the repair of inguinal and incisional hernias. Arch Surg. 1960; 81:847-54.

10. Falci F. Marlex mesh no tratamento da hérnia inguinal do adulto. O Hospital. 1969;73: 147-51.

11. Shulman AG, Amid PK, Lichtenstein IL. A survey of non-expert surgeons using the open tension-free mesh patch repair for primary inguinal hernias. Int Surg. 1995; 80(1):35-6.

12. Ger R. The management of certain abdominal herniae by intraabdominal closure of the neck of the sac. Preliminary communication. Ann R Coll Surg Engl. 1982; 64(5):342-4.

13. Toy FK, Smoot RT. Toy-Smoot laparoscopic hernioplasty. Surg Laparosc Endosc. 1991; 1(3):151-5.

14. Schultz L, Graber J, Pietrafitta J, Hickok D. Laser laparoscopic herniorraphy: a clinical trial preliminary results. J Laparoendosc Surg. 1990; 19(1): 41-5.

15. Roll S, Campos FG. Cirurgia laparoscópica. In: Silva AL, editor. Hérnias da parede abdominal. Clínica Brasileira de Cirurgia. São Paulo: Atheneu; 1997. p. 279-89.

16. Barbosa GA, Amaral VF, Lázaro da Silva A. Histologia dos sacos herniários das hérnias inguinais indiretas, diretas, recidivadas e encarceradas em adultos e crianças: identificação de fibras musculares. Rev Col Bras Cir. 2002; 29(1):1-11.

17. Silva AL. Estudo morfológico do saco herniário inguinal. Rev Hosp Clin Fac Med S Paulo. 1992; 42(1):65-8.

18. Silva AL. Uso do saco herniário no reforço da hernioplastia inguinal. Rev Col Bras Cir. 1995; 22(3):153-4.

19. Silva AL, Petroianu A. Próteses. In: Silva AL, editor. Hérnias. $1^{\text {a }}$ ed. São Paulo: Roca; 1992. p. 1127-37.

20. Falci F. Análise crítica das próteses na região inguinal. In: Silva AL, editor. Hérnias da parece abdominal. São Paulo: Atheneu;1997. p. 141-51.

21. Speranzini MB, Deutsch CR. Tratamento cirúrgico das hérnias das regiões inguinal e crural. São Paulo: Atheneu; 2001.

22. Lichtenstein IL, Shulman AG, Amid PK. The cause, prevention. and treatment of recurrent groin hernia. Surg Clin North Am. 1993 Jun;73(3):529-44

23. Speranzini MB, Deutsch CR. Avaliação dos resultados da correção das hérnias inguinais e crurais pelos métodos atuais. In: . Tratamento cirúrgico das hérnias das regiões inguinal e crural. Estado atual. São Paulo: Atheneu; 2002. p. 189208.

24. Wantz GE. Complications of synthetic protheses in hernia surgery. Probl Gen Surg. 1995; 12:79-83.

25. Stoppa RE. The preperitoneal approach and prosthetic repair of groin hernias". In: Nyhus LM, Condon Re, editors. Hernia. $4^{\text {th }}$ ed. Philadelphia: Lippincot; 1995. .p. 188-210.

26. Munshi IA, Wantz GE. Management of recurrent and perivascular femoral hernias by giant prosthetic reinforcement of the visceral sac. J Am Coll Surg. 1996; 182(5):417-22.

27. Taylor EW, Deware EP. Early return to work after repair of a unilateral inguinal hernia. Br J Surg. 1983; 70(10):599-600.

28. Bergstein JM, Condon RE. Obturador hernia: current diagnosis and treatment. Surgery. 1996; 119(2):133-6.

29. Eller R, Twaddell C, Poulos E, Jenevein E, McIntire D, Rissell S. Abdominal adhesions in laparoscopic hernia repair. Surg Endosc.1994; 8(3):181-4.

30. Silich RC, McSherry CK. Spermatic granuloma. An uncommon complication of the tension-free hernia repair. Surg Endosc. 1996; 10(5):537-9.

31. Hooker GD, Taylor BM, Driman DK. Prevention of adhesion formation with use of sodium hyaluronate-based bioresorbable membrane in a rat model of ventral hernia repair with polypropylene mesh: A randomized, controlled study. Surgery. 1996; 125(2):211-6.

32. Cheuvalier C, Hardy JC. Complication vésicale d'une cure de hernie par prothèse. Acta Urol Belg. 1987; 55(3):387-9.

33. Hamy A, Paineau J, Savigny JL, Vasse N, Visset J. Sigmoid perforation, an exceptional late complication of peritoneal prosthesis for treatment of inguinal hernia. Int Surg. 1997; 82(3):307-8

34. Hendrickse CW, Evans DS. Intestinal obstruction following laparoscopic inguinal hernia repair. Br J Surg. 1993; 80(11):1432.

35. Langer C, Neufang T, Kley C, Liersch T, Becker H. Central mesh recurrence after incisional hernia repair with Marlex-are the meshes strong enough? Hernia. 2001; 5(3):164-7.

36. Deysine M. Hernia repair with expanded polytetrafluoroethylene. Am J Surg. 1992; 163(4):422-4.

37. Klosterhalfen B, Klinge U, Schumpelick V. Functional and morphological evaluation of different polypropylene-mesh modifications for abdominal wall repair. Biomaterials. 1998;19(24):2235-46.

38. Franklin ME, Gonzalez JJ, Michaelson RP, Glass JL, Chock DA. Preliminary experience with new bioactive prosthetic material for repair of hernias in infected fields. Hernia. 2002; 6(4):1714. Epub 2002 Oct 19.

39. Weitzel SH, Botha AJ, Thomas PA. Late colocutaneous fistula after mesh repair of an inguinal hernia. Hernia. 1988; 2(1):39-40.

40. Leber FG, Garb MS, Alexander A, Reed WP. Long-term complications associated with prosthetic repair of incisional hernias. Arch Surg. 1988; 133(4): 378-82.

41. Morris-Stiff GJ, Hughts LE. The outcomes of nonabsorbable mesh placed within the abdominal cavity: literature review and clinical experience. J Am Coll Surg. 1998;186(3):352-67.

42. Goldenberg A, Matone J, Marcondes W, Focchi G. Efeitos da tela de polipropileno no testículo, epidídimo e ducto diferente de cães. Acta Cir Bras. 2001; 16(4):226-30.

43. Shin D, Lipshultz LI, Goldstein M, Barmé GA, Fuchs EF, Nagler HM, McCallum SW, Niederberger CS, Schoor RA, Brugh VM 3rd, Honig SC. Herniorraphy with polypropylene mesh causing inguinal vassal obstruction: a preventable cause of obstructive azoospermia. Ann Surg. 2005; 241(4):553-8. 
44. Shumpelick V. Does every hernia demand a mesh repair? Acritical review. Hernia. 2001; 5(1):5-8.

45. Nyhus LM. Ubiquitous use of prosthetic mesh in inguinal hernia repair: the dilemma. Hernia. 2000; 4(4):184-6.

46. Langer C, Neufang T, Kley C, Liersch T, Becker H. Central mesh recurrence after incisional hernia repair with Marlex - are the meshes strong enough? Hernia. 2001; 5(3):164-7.

47. Demirer S, Kepenecki I, Evirgen O, Birsen O, Tuzuner A, Karahuseyinoglu S, Ozban M, Kuterdem E. The effect of polypropylene mesh on ilioinguinal nerve in open mesh repair of groin hernia. J Surg Res. 2006; 131(2):175-81. Epub 2006 Jan 18.

48. Lichtenstein IL, Shulman AG, Amid PK, Montllor MM. Cause and prevention of postherniorrhaphy neuralgia: a proposed protocol for treatment. Am J Surg. 1988;155(6):786-90.

49. Laparoscopic versus open repair of groin hernia: a randomised comparison. The MRC Laparoscopic Groin Hernia Trial Group. Lancet. 1999; 54(9174):185-90.

50. Bay-Nielsen M, Perkins FM, Kehlet H; Danish Hernia Database. Pain and functional impairment 1 year after inguinal herniorrhaphy: a nationwide questionnaire study. Ann Surg. 2001; 233(1):1-7.

51. Kehlet H, Bay-Nielsen M, Kingsnorth A. Chronic postherniorrhaphy pain —a call for uniform assessment. Hernia. 2002; 6(4):178-81. Epub 2002 Sep 20.

52. Callesen J, Bech K, Nielsen R, Andersen J, Hesselfeldt P, Roikjær O, Kehlet H. Pain after groin hernia repair. Br J Surg. 1998; 85(10):1412-4.

53. Kumar S, Wilson RG, Nixon SJ, Macintyre IM. Chronic pain after laparoscopic and open mesh repair of groin hernia. Br J Surg. 2002; 89(11):1476-9.

54. Hay JM, Boudet MJ, Fingerhut A, Poucher J, Hennet H, Habib E, Veyrières M, Flamant Y. Shouldice inguinal hernia repair in the male adult: the gold standard? A multicenter controlled trial in 1.578 patients. Ann Surg. 1995; 222(6):719-27.

55. Minossi JG, Kemp R, Picanço HC et al. Avaliação pós-operatória tardia de pacientes submetidos a herniorrafia inguinocrural por via anterior, utilizando a técnica convencional ou a colocação de prótese sintética. ABCD Arq Bras Cir Dig. 2004; 17(4):163-9.

56. Luca-Filho CRP, Lopes-Filho GJ. Resultados precoces e tardios obtidos com a utilização da técnica de Lichtenstein nas hernioplastias inguinais em doentes portadores de hérnias dos tipos IIIA e IIIB de Nyhus. ABCD Arq Bras Cir Dig. 2004; 17(4):170-5.

57. Vironen J, Nieminen J, Eklund A, Paavolainen P. Randomized clinical trial of Lichtenstein patch or prolene hernia system for inguinal hernia repair. Br J Surg. 2006; 93(1):33-9.

58. Lázaro da Silva A, Souza PL. Complicações das próteses. In: Silva AL, editor. - Hérnias. $2^{a}$ ed. São Paulo: Roca; 2006. pp. 860-4.

59. Amid PK. Surgical treatment for postherniorrhaphy neuropathic inguinodynia: triple neurectomy with proximal end implantation. Contemp Surg. 2003; 59(6):276-80.

60. Casten DF. Functional anatomy of the groin area as related to the classification and treatment of groin hernia. Am J Surg. 1967; 114(6):894-9.

61. Halverson K, Mc Vay C. Inguinal and femoral hernioplasty. Arch Surg. 1970; 101(2):127-35.

62. Nyhus L. Complications of groin hernia repair. Audio Digest Gen Surg. 1995; 1291):7-12.

63. Gilbert AI. An anatomic and functional classification for the diagnosis and treatment of inguinal hernia. Am J Surg. 1989; 157(3):331-3.
64. Rutkow JM, Robbins AW. Aspectos demográficos classificatórios e sócio-econômicos do reparo herniário nos Estados Unidos da América. Clin Cir Am Norte. 1993; 3: 443-8.

65 . Bendavid R. The rational use of mesh in hernias. A perspective. Int Surg. 1992;77(4): 229-31.

66. Nyhus LM, Donahve PE. Groin hernia: past, present and future. Probl Gen Surg. 1995. 12(1):7-12.

67. Nyhus LM. Comentário ao artigo de Mc Gillicuddy, 1998.

68. Kingsnorth NA, Gray MR, Nott DM. Prospective randomized trial comparing the Shouldice technique and plication darn of inguinal hernia. Br J Surg. 1992; 79(10):1068-70.

69. Kux M, Fuchsjäger N, Schemper M. Shouldice is superior to Bassini inguinal herniorraphy. Am J Surg. 1994; 168(1):15-8.

70. Paul A, Troidl H, Williams JI, et al. Randomized trial of modified Bassini versus Shouldice inguinal hernia repair. The Cologne Hernia Study Group. Br J Surg. 1994; 81(10):1531-4.

71. Mittelstaedt WEM, Rodrigues AJJr, Duprat J et al.Tratamento das hérnias inguinais: Bassini ainda atual? Estudo randomizado, prospectivo e comparativo entre três técnicas operatórias: Bassini, Shouldice, Mc Vay. Rev Ass Med Bras. 1999; 45(2):105-14.

72. Abrahamson J. Factors and mechanisms hading to recurrence. Probl Gen Surg. 1995; 12(1):55-65.

73. Friis G, Lindahl F. The tension-free hernioplasty in a randomized trial. Am J Surg. 1996; 172(4):315-9.

74. Lex A, Valtorth A. Hérnia. Aspecto clínico e cirúrgico. São Paulo: Panamed; 1984. p. 227.

75. Cray MR, Curtis JM, Elkington JS. Colovesical fistula after laparoscopic inguinal hernia repair. Br J Surg. 1994; 81(8):1213-4.

76. Hamy A, Paineau J, Savigny JL, et al. Sigmoid perforation, an exceptional late complication of peritoneal prosthesis for treatment of inguinal hernia. Int Surg. 1997; 82(3):307-8.

77. Tsang S, Normand R, Karlin R. Small bowel obstruction: a morbid complication after laparoscopic herniorrhaphy. Am Surg. 1994; 60(5):333-4.

78. Silva AL. Cirurgia laparoscópica para hérnias inguinais [editorial]. Rev Col Bras Cir. 1992; 19(6): III.

79. Rahal F. Importância da cirurgia das hérnias no aprendizado de cirurgia. Bol Inf Col Bras Cir. 1997; 97(1):45-6.

80. Falci F. Uso de prótese no reforço do triângulo inguinal. In: Silva AL, editor. Hérnias. $2^{a}$ ed. São Paulo: Roca; 2006. p. 532-7.

81. Silva AL. Tratamento do saco herniário. In: Silva A, editor. Hérnias. $2^{a}$ ed. São Paulo: Roca; 2006. p. 530-1.

82. Melo RM. Bases do tratamento da hérnia inguinal. In: Silva AL, editor. Hérnia. $2^{a}$ ed. São Paulo: Roca; 2006; p. 534-8.

83. Nyhus LM. Individualization of hernia repair: a new era. Surgery. 1993; 114(1): 1-2.

84. Minossi JG, Lázaro da Silva A. Aspectos médicos-legais da cirurgia para hérnia inguinal. Rev Col Bras Cir. 2005; 32(3): 214-7.

85. Ferrari W . Tratamento das grandes hérnias do abdomen. Rev Med Est Guanabara. 1963; 30: 59 - 115.

86. Ferrari W . Restauração cirúrgica da parede abdominal incompetente. J Bras Méd. 1968; 14: 349 - 79.

87. Almeida AD, Lázaro da Silva A, Goffi FS. Laparotomias. In: Goffi FS, editor. Técnica cirúrgica. Bases anatômicas, fisiopatológicas e técnicas da cirurgia. $4^{\mathrm{a}}$ ed. São Paulo: Atheneu; 1996. p. 456-64.

88. Silva AL, Guerra AJ, Castro MAM. Hérnia incisional. In: Silva AL, editor. Hérnias. $2^{a}$ ed. São Paulo: Roca; 2006. p. 398-420. 
89. Silva AL. Emprego do saco herniário na correção cirúrgica da hérnia incisional. In: Silva AL, editor. Hérnias. $2^{a}$ ed. São Paulo: Roca; 2006. p. 420-1.

Como citar este artigo:

Minossi JG, Silva AL, Spadella CT. O uso da prótese na correção das hérnias da parede abdominal é um avanço, mas o seu uso indiscriminado, um abuso. Rev Col Bras Cir. [periódico na Internet] 2008; 35(6). Disponível em URL: http://www.scielo.br/rcbc
Endereço para Correspondência:

José Guilherme Minossi

Praça Irmãos Ferreira, 171

18760-000 - CERQUEIRA CÉSAR - SP

E-mail:jminossi@uol.com.br ou jminossi@fmb.unesp.br 